\title{
Géochimie et distribution de métaux à l'intérieur de carottes de sédiment prises du lac Saint-Louis (fleuve Saint-Laurent) et datées par radioactivité Geochemistry and distribution of metals in radiodated sediment cores from Lake St. Louis, St. Lawrence River
}

\author{
A. Mudroch et S. R. Joshi
}

Volume 4, numéro 1, 1991

URI : https://id.erudit.org/iderudit/705087ar

DOI : https://doi.org/10.7202/705087ar

\section{Aller au sommaire du numéro}

\section{Éditeur(s)}

Université du Québec - INRS-Eau, Terre et Environnement (INRS-ETE)

ISSN

0992-7158 (imprimé)

1718-8598 (numérique)

Découvrir la revue

Citer cet article

Mudroch, A. \& Joshi, S. R. (1991). Géochimie et distribution de métaux à

l'intérieur de carottes de sédiment prises du lac Saint-Louis (fleuve

Saint-Laurent) et datées par radioactivité. Revue des sciences de l'eau / Journal of Water Science, 4(1), 1-17. https://doi.org/10.7202/705087ar

\section{Résumé de l'article}

La distribution de la grosseur des particules, la composition géochimique et la concentration en métaux ( $\mathrm{Cu}, \mathrm{Cr}, \mathrm{Pb}, \mathrm{Co}, \mathrm{V}, \mathrm{Ni}, \mathrm{Hg}$ et $\mathrm{Zn}$ ) furent déterminés dans des carottes datées (à l'aide de matériel radioactif) provenant du lac Saint-Louis et du fleuve Saint-Laurent. Environ $20 \mathrm{~cm}$ de sédiments fins se sont accumulés durant les 30 dernières années à deux endroits situés à proximité du centre du lac. Par contre, une couche de sédiment de $20 \mathrm{~cm}$ d'épaisseur s'est déposée pendant les cinq dernières années à deux différents endroits de la partie sud du lac. Les profils de concentration des éléments majeurs et de la plupart des métaux présents dans les carottes de sédiment indiquent un apport continu de matériel de nature géochimique semblable aux aires d'échantillonnage. Une distribution uniforme de la grosseur des particules à l'intérieur du profil de sédiment montre que ces aires constituent des zones de dépôt pour des particules de propriétés hydrodynamiques semblables que transportent les eaux du fleuve. Les profils de concentration du mercure $(\mathrm{Hg})$ et du zinc (Zn) dans les sédiments sont significatifs d'une charge provenant d'une source locale située sur le lac Saint-Louis. Les concentrations en métaux mesurées dans les sédiments du lac Saint-Louis dépendent du tri des particules, du transport et du dépôt des particules de la fraction limoneuse (taille comprise entre $2 \mu \mathrm{g}$ et $63 \mu \mathrm{g}$ ) qui peuvent contenir de plus faibles concentrations en métaux comparativement aux particules de grosseur comparable à l'argile (taille $<2 \mu \mathrm{g}$ ) qui caractérisent les bassins de dépôt du lac Ontario. 


\title{
Géochimie et distribution de métaux à l'intérieur de carottes de sédiment prises du lac Saint-Louis (fleuve Saint-Laurent) et datées par radioactivité
}

\author{
Geochemistry and distribution of metals \\ in radiodated sediment cores \\ from Lake St. Louis, \\ St. Lawrence River
}

A. MUDROCH1 , S.R. JOSHI

Reçu le 5 juillet 1988, accepté pour publication le 12 juin $1990^{*}$

RÉSUMÉ

La distribution de la grosseur des particules, la composition géochimique et la concentration en métaux ( $\mathrm{Cu}, \mathrm{Cr}, \mathrm{Pb}, \mathrm{Co}, \mathrm{V}, \mathrm{Nl}, \mathrm{Hg}$ et $\mathrm{Zn}$ ) furent déterminés dans des carottes datées (a l'aide de matériel radioactif) provenant du lac Saint-Louls et du fleuve Saint-Laurent. Environ $20 \mathrm{~cm}$ de sédiments fins se sont accumulés durant les $\mathbf{3 0}$ dernières années à deux endroits situés à proximité du centre du lac. Par contre, une couche de sédiment de $20 \mathrm{~cm}$ d'épaisseur s'est déposée pendant les cinq dernières années à deux différents endrolts de la partie sud du lac. Les prolits de concentration des éléments majeurs et de la plupart des métaux présents dans les carottes de sédiment Indiquent un apport continu de matériel de nature géochimique semblable aux aires d'6chantilionnage. Une distribution uniforme de la grosseur des particules à l'intérieur du profil de sédiment montre que ces aires constituent des zones de dépót pour des particules de propriétés hydrodynamiques semblables que transportent les eaux du fieuve. Les profils de concentration du mercure $(\mathrm{Ho})$ et du zinc $(\mathrm{Zn})$ dans les sédiments sont significatifs d'une charge provenant d'une source locale située sur le lac Saint-Louls. Les concentrations en métaux mesurées dans les sédiments du lac Saint-Louis dépendent du tri des particules, du transport et du dépót des particules de la fraction limoneuse (taille comprise entre $2 \mu \mathrm{g}$ et $63 \mu \mathrm{g}$ ) qui peuvent contenir de plus faibles concentrations en métaux comparativement aux particules de grosseur comparable à l'argile (tailie $<2 \mu \mathrm{g}$ ) qui caractérisent les bassins de dépôt du lac Ontario.

Mots clés : Sédiment, datation, géochimie, métaux.

1. Direction de la recherche sur les lacs, Institut national de recherche sur les eaux, 867 Lakeshore Road, P.0. Box 5050, Burlington (Ont), Canada, L7R 4A6.

- Les commentaires seront reçus jusqu'au 30 octobre 1991. 
Particle size distribution, geochemical composition and concentrations of metals ( $\mathrm{Cu}, \mathrm{Cr}, \mathrm{Pb}, \mathrm{Co}, \mathrm{V}, \mathrm{Ni}, \mathrm{Hg}$ and $\mathrm{Zn}$ ) were determined in radiodated cores collected from Lake St. Louis, St. Lawrence River, Quebec, Canada, to assess the significance of the lake as a sink of contaminants transported through the St. Lawrence River. The cores were collected at four stations in the depositional areas identified by earlier surveys of Lake St. Louis. Stations Nos. 3 and 9 were located in the northem part of the lake, and stations 17 and 21 along the southern shore. A modified Kajak-Brinkhurst corer was used to collect the sediment cores. The concentrations of major elements and metals were determined by $X$-ray filuorescence spectrometry. The concentrations of $\mathrm{Hg}$ and inorganic $\mathrm{C}$ were determined by cold vapour atomic absorption spectrometry and Leco carbon analyzer, respectively. The total $\mathrm{Pb}-210$ and Ra-226 levels in one centimeter sediment core sections were measured by low-energy gamma-ray spectrometry for calcutation of sediment age. The effects of mixing on sediment age has been estimated.

About $20 \mathrm{~cm}$ of fine grained sediment accumulated during the past 30 years at two sites near the centre of the lake. However, a $20 \mathrm{~cm}$ thick sediment layer was deposited over the past five years at two different areas in the southern part of the lake. Calculated sedimentation rate for surface sediments were 207, 296,1837 , and $2912 \mathrm{mg} \mathrm{cm}^{-2} \mathrm{r}^{-1}$ for stations $3,9,17$, and 21, respectively. The concentration profiles of ten major elements (Si, Al, Fe, Ca, Mg, $\mathrm{Na}, \mathrm{K}, \mathrm{Ti}, \mathrm{P}$, and $M n$ ), organic and inorganic $C$ and most of the investigated metals were similar and consistent in the sediment profiles at all stations, and indicated a continuous input of geochemically similar material at the sampling sites.

A uniform particle size distribution within the sediment profile showed that these areas are settling zones for particles of simllar hydrodynamic properties transported by the St. Lawrence River. The concentration profiles of $\mathrm{Hg}$ in the cores indicated a contlnuous input of $\mathrm{Hg}$ along the southern shore of the lake. The concentration profile of $\mathrm{Hg}$ in the core from the northern part of the lake had a similar pattern to that from Lake St. Clair and Lake Ontario, showing a decrease of $\mathrm{Hg}$ in the surficial sediments. This decrease corresponds to implemented control of $\mathrm{Hg}$ losses by industry in Canada and the USA over the past 15 years. The concentration profiles of $\mathrm{Hg}$ and $\mathrm{Zn}$ in the sediments indicated an Input from local sources along Lake St. Louis.

The concentrations of $\mathrm{Cr}, \mathrm{V}$ and $\mathrm{Co}$ in sediments in the depositional zones were similar to both background and surficial sediment concentrations in depositional basins of Lake Ontario. The concentrations of $\mathrm{Cu}$, $\mathrm{Ni}$ and $\mathrm{Pb}$ were lower than those in surficial sediments in Lake Ontario depositional basins. Observed concentrations of investigated metals in Lake St. Louis sediments were ascribed to the particle sorting, transport and deposition of silt-size ( 2 to $63 \mathrm{\mu m}$ grain size) particles which may contain smaller concentrations of metals than clay-size (< $2 \mu \mathrm{m}$ grain size) particles in the depositional basins of Lake Ontario. It was concluded that Lake St. Louis provides only a temporal storage for metals associated with clay-size sediment particles which may eventually become resuspended and moved downstream.

Key-words : Sediment, dating, geochemistry, metals.

* Original English version of this paper is available. Please address your request to: Revue des sciences de l'eau, INRS-Eau, 2800 rue Einstein, Québec (Qué), Canada GIX 4N8. 


\section{INTRODUCTION}

Le fleuve Saint-Laurent, avec un débit moyen de $7700 \mathrm{~m}^{3} / \mathrm{s}$ à la source et de $12000 \mathrm{~m}^{3} / \mathrm{s}$ à l'embouchure, draine les Grands Lacs vers le golfe Saint-Laurent de l'océan Atlantique. Le fleuve constitue un système d'accumulation et de transport de contaminants toxiques. Certains de ces composés chimiques toxiques proviennent du lac Ontario, mais plusieurs autres ont pour origine des sources diffuses et ponctuelles réparties le long du fleuve (ALLAN, $1986 ; 1987$ ). Les contaminants solubles dans l'eau et les contaminants particulaires provenant a la fois du lac Ontario et de sources bordant le fleuve sont transportés par celui-ci jusqu'à son estuaire.

Le lac Saint-Louis, avec une superficie de $148 \mathrm{~km}^{2}$ (PRÉfONTAINE, 1942), est l'un des quatre petits lacs riverains qui font intégralement partie du fleuve Saint-Laurent (fig. 1). Le lac est situé au confluent de la rivière des Outaouais et du fleuve Saint-Laurent. Les profondeurs moyenne et maximale du lac sont respectivement de $2 \mathrm{~m}$ et $28 \mathrm{~m}$ (PAGEAU, 1971). La distribution spatiale de métaux et de BPC dans les sédiments de surface du lac Saint-Louis a été étudiée par JARRY et al. (1985). Les résultats de cette recherche ont montré que les concentrations en contaminants dans les sédiments surpassaient les normes actuelles du ministère de l'Environnement de l'Ontario concernant les rejets de matériaux de dragage en eaux libres.

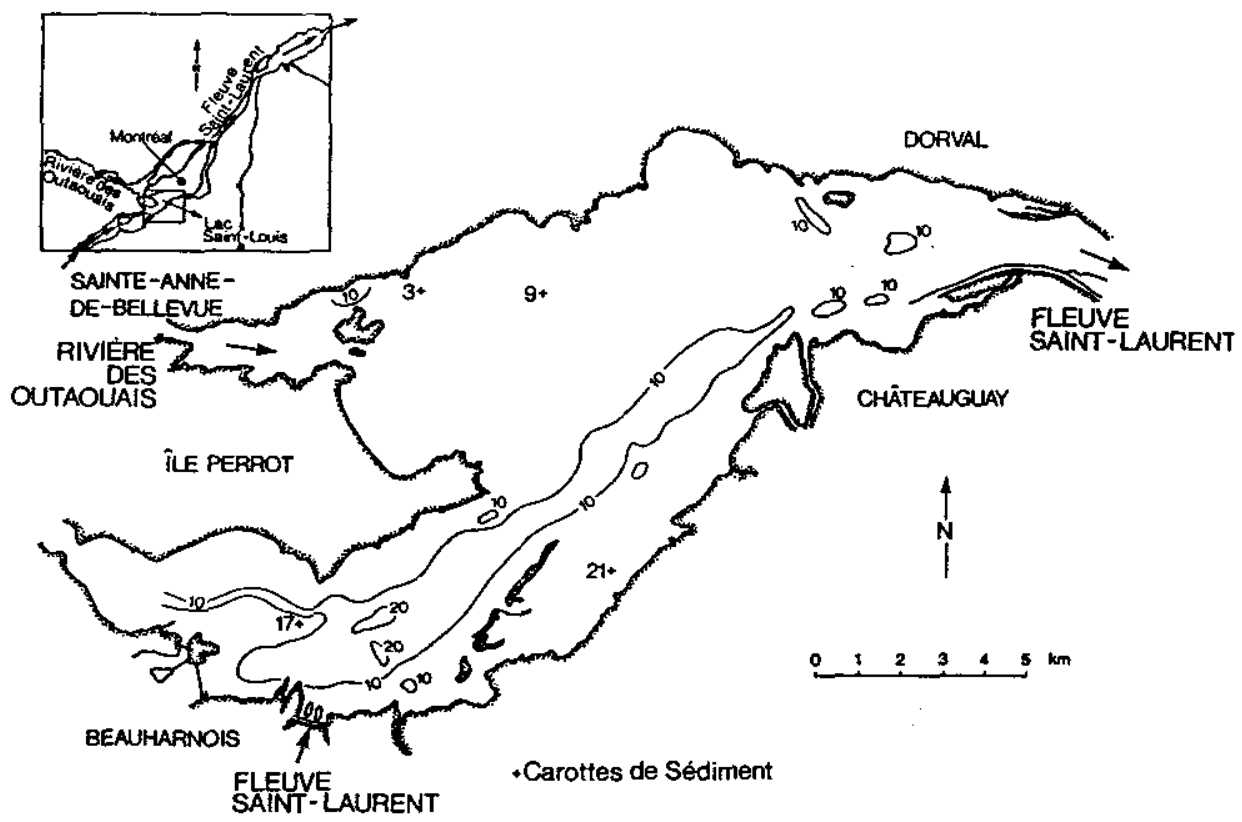

Figure 1 Stations d'échantillonnage du lac Saint-Louis.

Sampling stations in Lake St. Louis. 
Les objectifs de la présente étude étaient d'observer les variations temporelles dans la géochimie des sédiments ainsi que l'évolution historique des concentrations en contaminants dans les sédiments, afin d'évaluer limportance du lac Saint-Louis comme source de contaminants transportés par le fleuve Saint-Laurent.

\section{MATÉRIEL ET MÉTHODES}

\section{Cueillette des sédiments et préparation des échantillons}

Le sédiment a été recueilli dans les zones de dépôt identifiées par des relevés antérieurs du lac Saint-Louis (JARRY et al., 1985 ; RUKAVINA, 1986). Quatre sites ont été choisis par le carottage dans le lac (fig. 1). Trois carottes ont été prélevées pour chacune des stations d'échantillonnage en utilisant un échantillonneur Kajak-Brinkhurst modifié et équipé d'un recouvrement intérieur en plastique. L'échantillonneur est une combinaison d'un instrument Kajak-Brinkhurst et d'un échantillonneur à gravité Benthos, doté d'une valve automatique Benthos modifiée (MAWHINNEY et BISUTTI, 1987). Toutes les carottes échantillonnées ont été subdivisées en sections de $1 \mathrm{~cm}$. Toutes les sections provenant de deux carottes pour chacune des stations ont été lyophilisées, broyées pour obtenir des particules de taille d'environ $190 \mu \mathrm{m}$ et utilisées pour la datation et les analyses géochimiques. Les sections des troisièmes carottes ont été utilisées pour la détermination de la distribution de la taille des particules.

\section{Méthodes d'analyse}

La détermination de la distribution de la taille des particules a été effectuée par la méthode décrite par DUNCAN et LAHAIE (1979). Les concentrations des éléments majeurs ( $\mathrm{Si}, \mathrm{Al}, \mathrm{Fe}, \mathrm{Ca}, \mathrm{Mg}, \mathrm{K}, \mathrm{Na}, \mathrm{Ti}, \mathrm{P}$ et $\mathrm{Mn}$ ) et des métaux (Co, $\mathrm{Cu}, \mathrm{Pb}, \mathrm{V}, \mathrm{Ni}$ et $\mathrm{Zn}$ ) ont été déterminées par spectométrie de fluorescence aux rayons $X$ en utilisant la méthode décrite par MUDROCH (1985). Les concentrations en $\mathrm{Hg}$ ont été déterminées dans tous les échantillons par la méthode décrite par MUDROCH et KOKOTICH (1987). Les concentrations en C organique et inorganique ont été déterminées à l'aide d'un analyseur de carbone Leco.

\section{Datation des sédiments}

Les niveaux totaux de $\mathrm{Pb}-210$ et de Ra-226 dans les sections des carottes de sédiment ont été mesurés par spectrométrie aux rayons gamma de basse énergie en utilisant les techniques décrites par JOSHI (1987). La contribution du Pb-210 en excès ou provenant de l'atmosphère (contribution que l'on notera simplement $\mathrm{Pb} / 210$ par la suite) a été obtenue en soustrayant la quantité de $\mathrm{Pb}-210$ supportée par le Ra-226 de la quantité totale de $\mathrm{Pb}-210$. 


\section{Calcul de l'âge des sédiments}

En considérant un taux d'apport constant en $\mathrm{Pb}-210$, on peut montrer (GOLDBERG, 1963) que l'âge $t$ (en années) d'une section de carotte de sédiment localisée à une profondeur de $\mathrm{x} \mathrm{cm}$ sous l'interfac eau-sédiment est donné par :

$$
t=\frac{1}{\lambda} \quad \ln \frac{A(0)}{A(x)}
$$

où $A(0)$ représente la quantité totale de $\mathrm{Pb}-210(\mathrm{~Bq} \mathrm{~cm}-2)$ dans la cclonne de sédiment, $A(x)$ est la quantité de $\mathrm{Pb}-210$ sous les sédiments d'âge $t$, et $\lambda$ est la constante de désintégration du Pb-210 évaluée à $0,0311 \mathrm{a}^{-1}$. Les activités $A(0)$ et $A(x)$ ont été estimées selon la description donnée par JOSH et al. (1988), qui ont récemment mis en évidence les procédures applicables pour l'estimation de ces paramètres lorsque les mesures de Pb-210 ne sont disponibles que pour une profondeur limitée, comme cela est le cas dans la présente étude. L'effet du mélange sur l'âge des sédiments a été estimé en appliquant l'équation suivante (OLDFIELD et APPLEBY, 1984) :

ois

$$
\begin{gathered}
t=\frac{1}{\lambda} \quad \frac{A(0)}{\ln \frac{A(x)+A(m)}{A(x)} \cdot A_{m}(0)} \\
A_{m}=\frac{C(x)}{C(s)}
\end{gathered}
$$

Dans cette équation, $A_{m}(0)$ représente l'activité intégrée du $\mathrm{Pb}-210$ dans la zone de mélange, $\mathrm{C}(\mathrm{s})$ est la concentration en $\mathrm{Pb}-210$ sous la zone de mélange $\left(\mathrm{Bq} \mathrm{g}^{-1}\right)$ et $\mathrm{C}(\mathrm{x})$ est la concentration en $\mathrm{Pb}-210$ à une profondeur $x\left(B q g^{-1}\right)$.

\section{Résultats}

Toutes les carottes de sédiment étaient constituées d'argile limoneuse grise avec des particules de $63 \mu \mathrm{m}$. En général, $54 \%$ à $77 \%$ des particules étaient de $8 \mu \mathrm{m}$, et $21 \%$ à $46 \%$ étaient de $2 \mu \mathrm{m}$. Des carottes d'environ $20 \mathrm{~cm}$ à $24 \mathrm{~cm}$ de longueur ont été récupérées aux stations 21 et 17 . Le sédiment obtenu en deçà de cette profondeur était un mélange de sable fin et d'argile. Des carottes plus longues (jusqu'à $30 \mathrm{~cm}$ ) ont été recueillies aux stations 3 et 9 . Le sédiment obtenu sous la profondeur de $30 \mathrm{~cm}$ était constitué d'argile fine et ferme.

Les profils âge/profondeur de quatre carottes de sédiment du lac SaintLouis, obtenus à partir des équations 1 et 2, sont présentés à la figure 2. Les âges des sédiments ont été obtenus à partir du taux de sédimentation calculé pour chacun des sous-échantillons, soit chacune des sections de carotte de sédiment. Toutefois, les taux de sédimentation pour les sections de sédiment de surface étaient de 207, 296, 1837 et $2912 \mathrm{mg} \mathrm{cm}^{-2} \mathrm{a}^{-1}$ pour les stations 3 , 9,17 et 21 , respectivement. La zone de mélange dans la carotte 21 n'a pu être établie à partir du profil mesuré du $\mathrm{Pb}-210$ et, par conséquent, aucun âge influencé par le mélange n'a été assigné aux sections de carottes prélevées. 
L'épaisseur estimée de la zone de mélange dans les carottes 3, 9 et 17 était de $5,3,6,2$ et $4,5 \mathrm{~cm}$, respectivement. Dans ces carottes, l'utilisation d'un paramètre de mélange conduit à un estimé plus bas de l'âge. Ceci est en accord avec les prédictions théoriques (JOSHI et al., 1988). Les résultats indiquent que le sédiment déposé aux stations 17 et 21 est très récent.

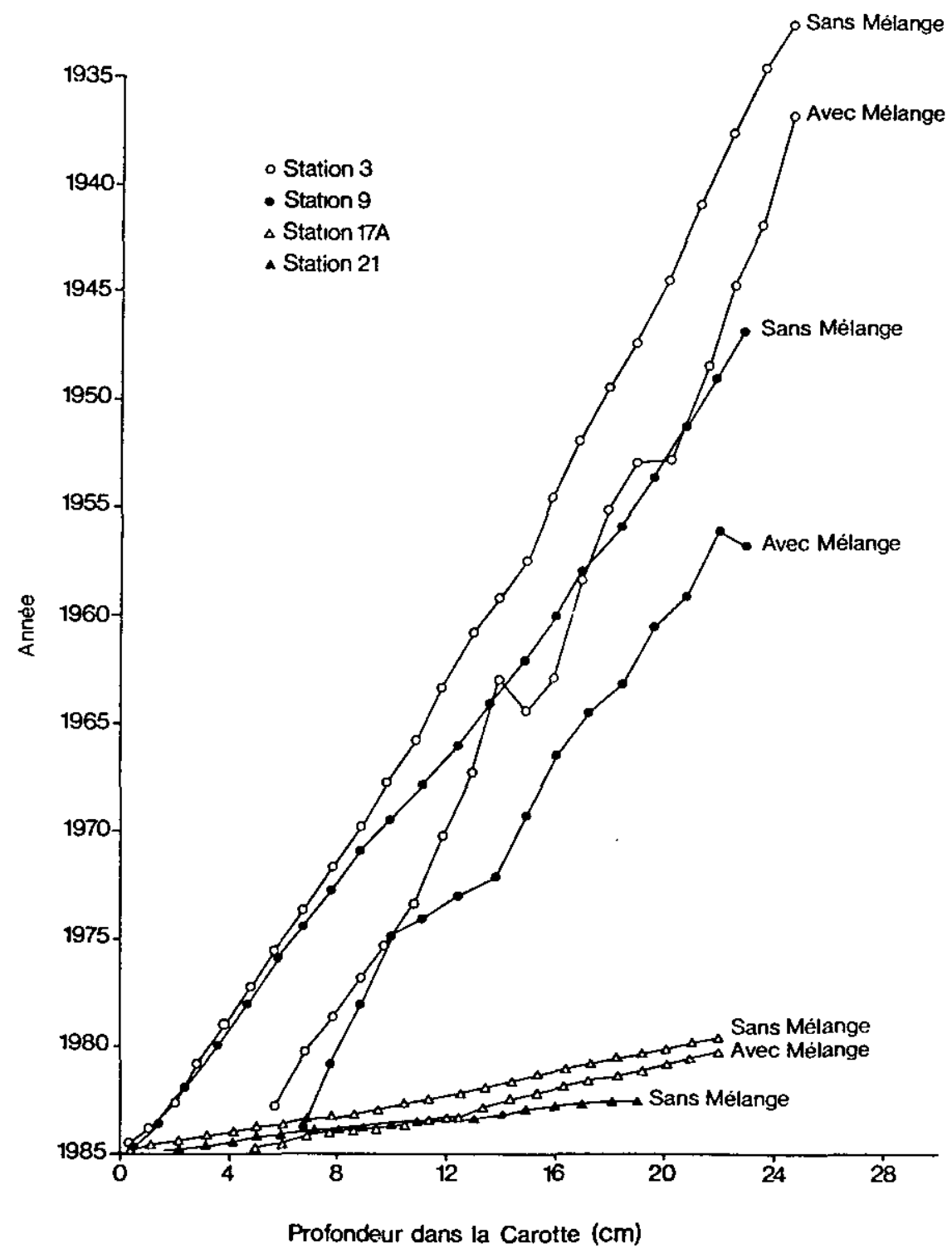

Figure 2 Profils áge/profondeur des carottes de sédiment.

The age/depth profiles of sediment cores. 
En général, des concentrations similaires en éléments majeurs et en C organique et inorganique ont été mesurées dans les profils de sédiment de toutes les stations. En conséquence, seuls les profils de concentration de ces éléments pour la carotte 3 sont illustrés (fig. 3). Les profils de concentration pour $\mathrm{Cu}, \mathrm{Ni}, \mathrm{Co}, \mathrm{Pb}, \mathrm{Cr}, \mathrm{Zn}$ et $\mathrm{V}$ dans les sédiments des stations 3,17 et 21 sont donnés aux figures 4 à 6 , et ceux pour $\mathrm{Hg}$ dans les sédiments des stations 3 et 21 , à la figure 7. L'âge des sédiments montré dans chacun des cas est celui obtenu par l'incorporation du paramètre de mélange, à l'exception de la station 21 où, tel que souligné précédemment, la profondeur de mélange n'a pu être établie.

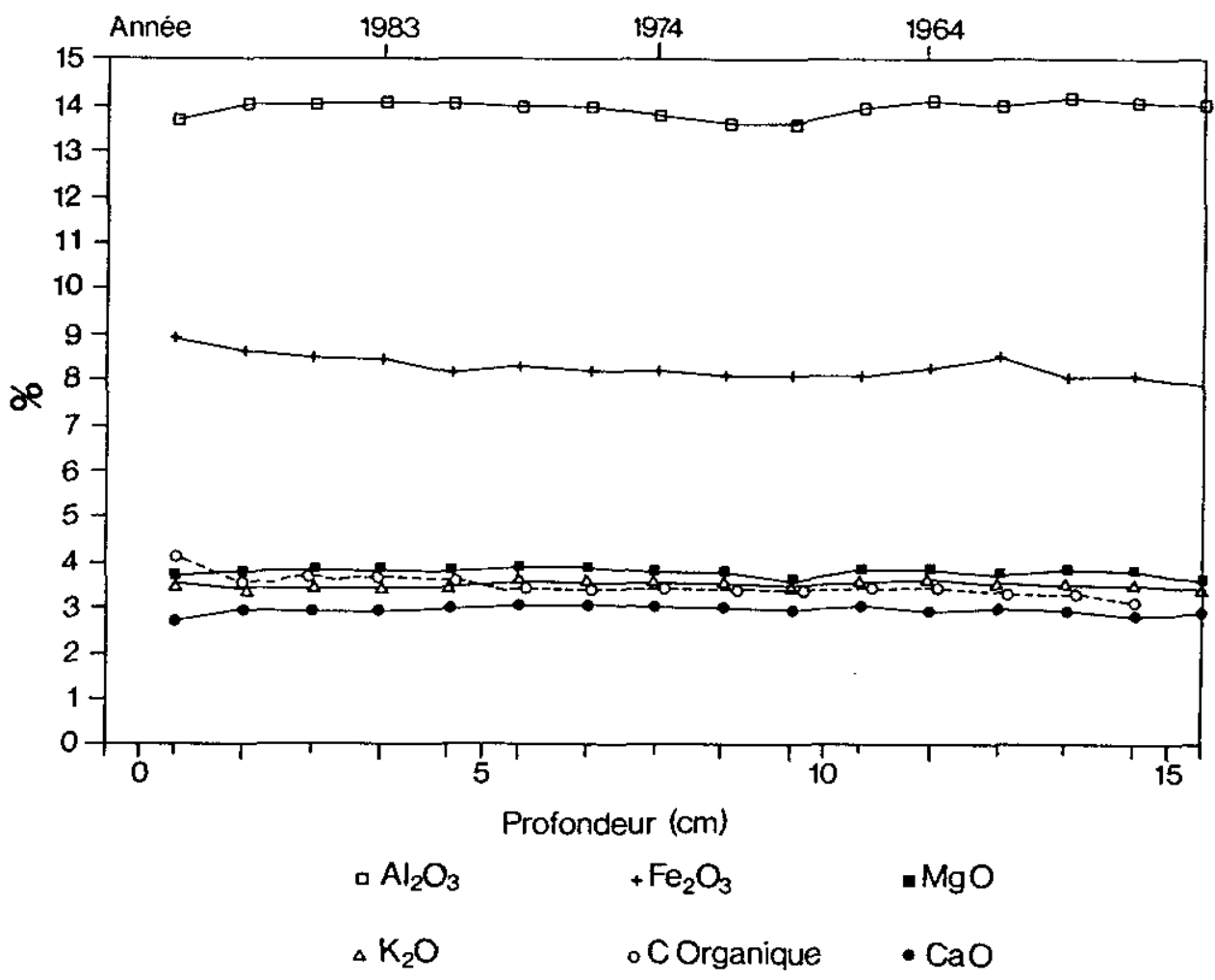

Figure 3 Profils de concentration des éléments majeurs dans les sédiments de la station 3.

Concentration profiles of major elements in the sediment from station 3. 


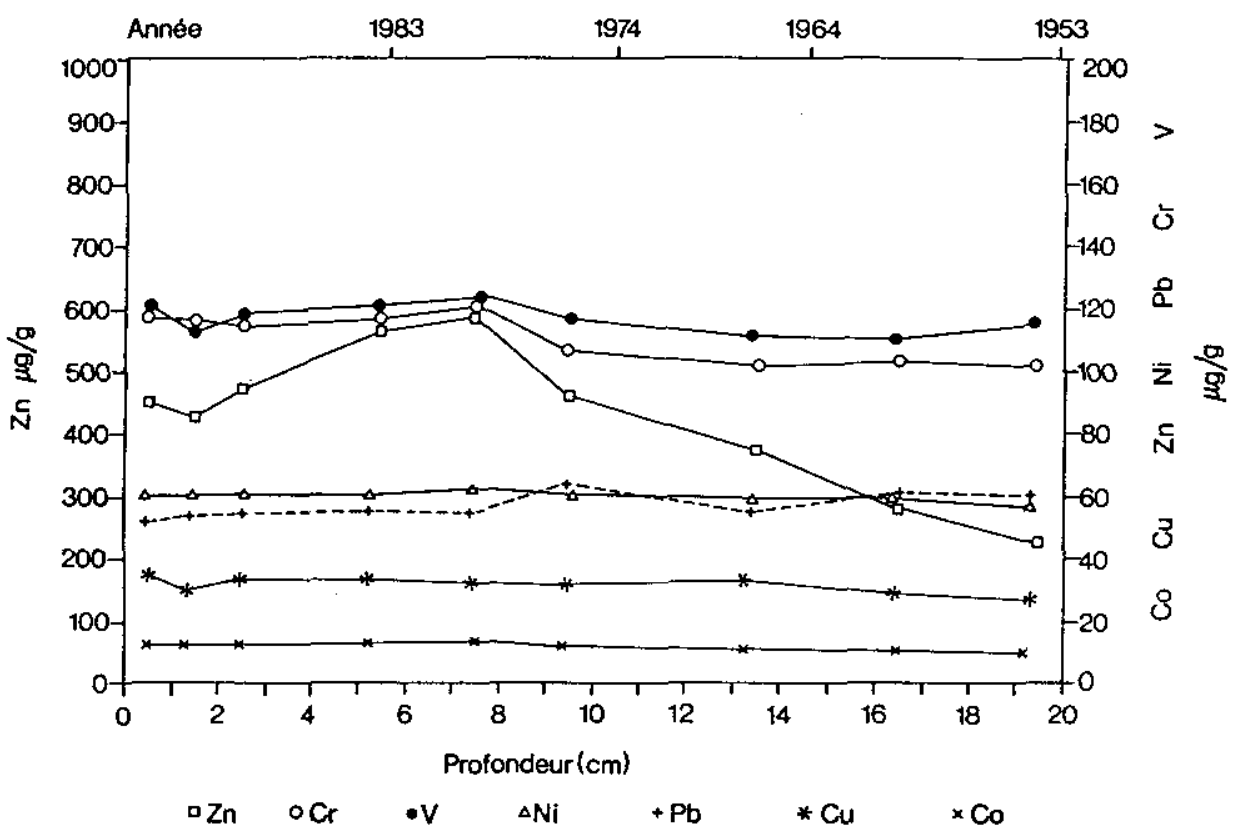

Figure 4 Profils de concentration des métaux dans les sédiments de la station 3. Concentration profiles of metals in sediment from station 3.

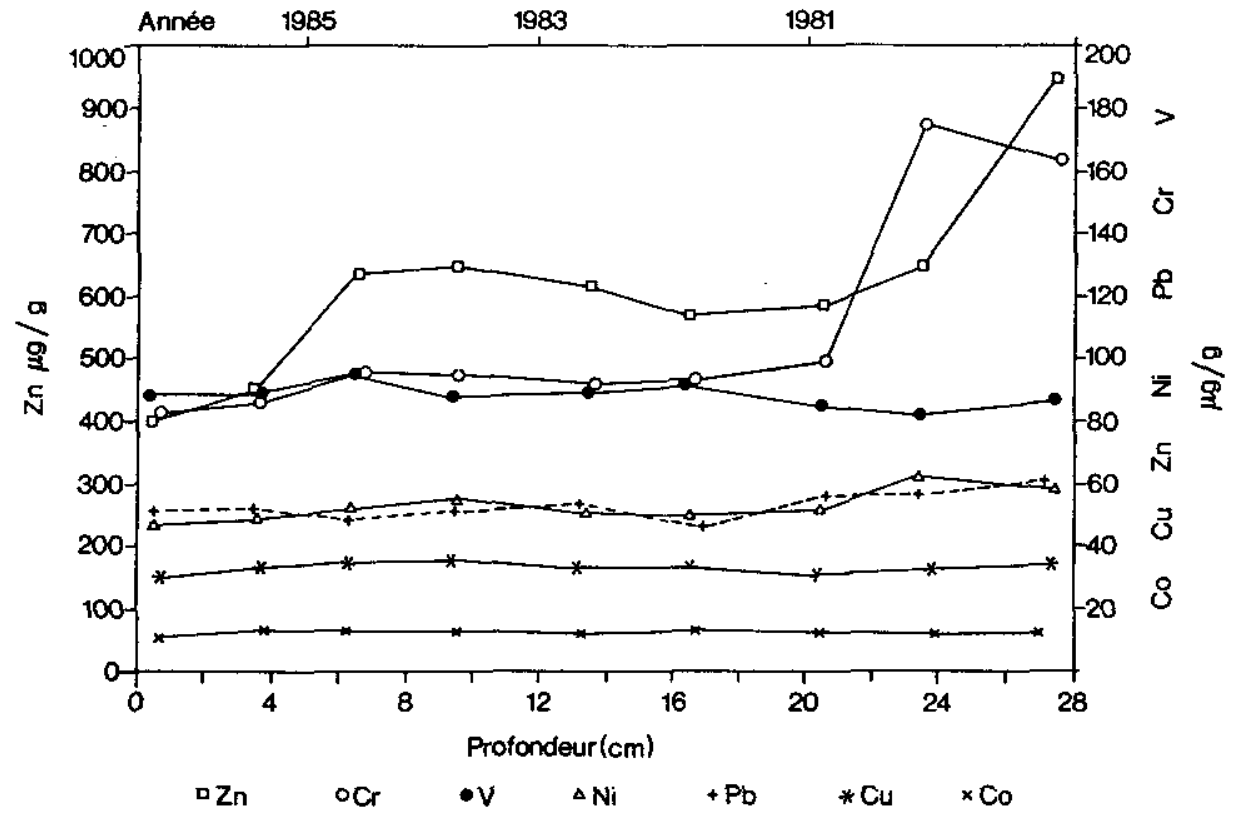

Flgure 5 Profils de concentration des métaux dans les sédiments de la station 17. Concentration profiles of metals in sediment from station 17. 


\section{DISCUSSION}

La datation des carottes de sédiment a montré que $20 \mathrm{~cm}$ de sédiment récent s'est déposés durant les cinq dernières années aux stations 17 et 21 . L'incorporation du paramètre de mélange dans le calcul de l'áge du sédiment a montré qu'environ $4,5 \mathrm{~cm}$ du sédiment de la station $21 \mathrm{n}$ 'a pu être déterminée à partir du profil mesuré de Pb-210. Il semble bien que ces zones dans le lac Saint-Louis ne sont que des zones de transition pour le dépôt de grains fins de sédiments. D'autre part, $20 \mathrm{~cm}$ de sédiment récent aux stations 3 et 9 s'est déposé durant les 30 dernières années, ce qui indique que ces régions du lac sont les zones de dépót pour une partie des grains fins de sédiments (taille des grains $<63 \mu \mathrm{m}$ ) transportés par le fleuve.

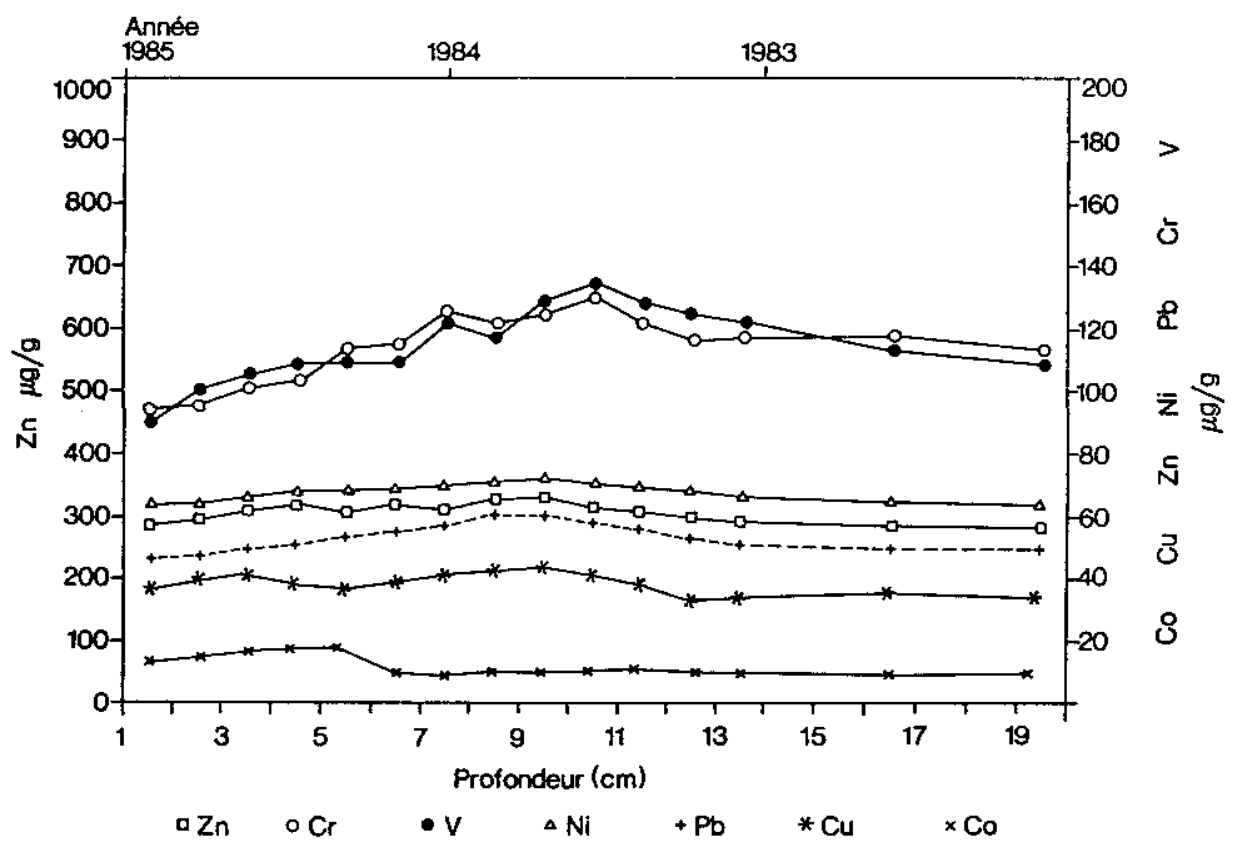

Figure 6 Profils de concentration des métaux dans les sédiments de la station 21.

Concentration profiles of metals in sediment from station 21.

Les profils de concentration des éléments majeurs et du carbone inorganique (ce dernier représentant les concentrations en carbonates) ont indiqué un apport continu de matière géochimique similaire au cours des 40 dernières années aux stations 3 et 9 . Seules de petites variations ont eu lieu dans l'accumulation des métaux associés aux sédiments de l'une ou lautre de ces deux stations. Une distribution uniforme de la taille des particules et une géochimie semblable à l'intérieur de la couche de $30 \mathrm{~cm}$ de sédiment à la station 3 ont confirmé que cette région est une zone de sédimentation pour 


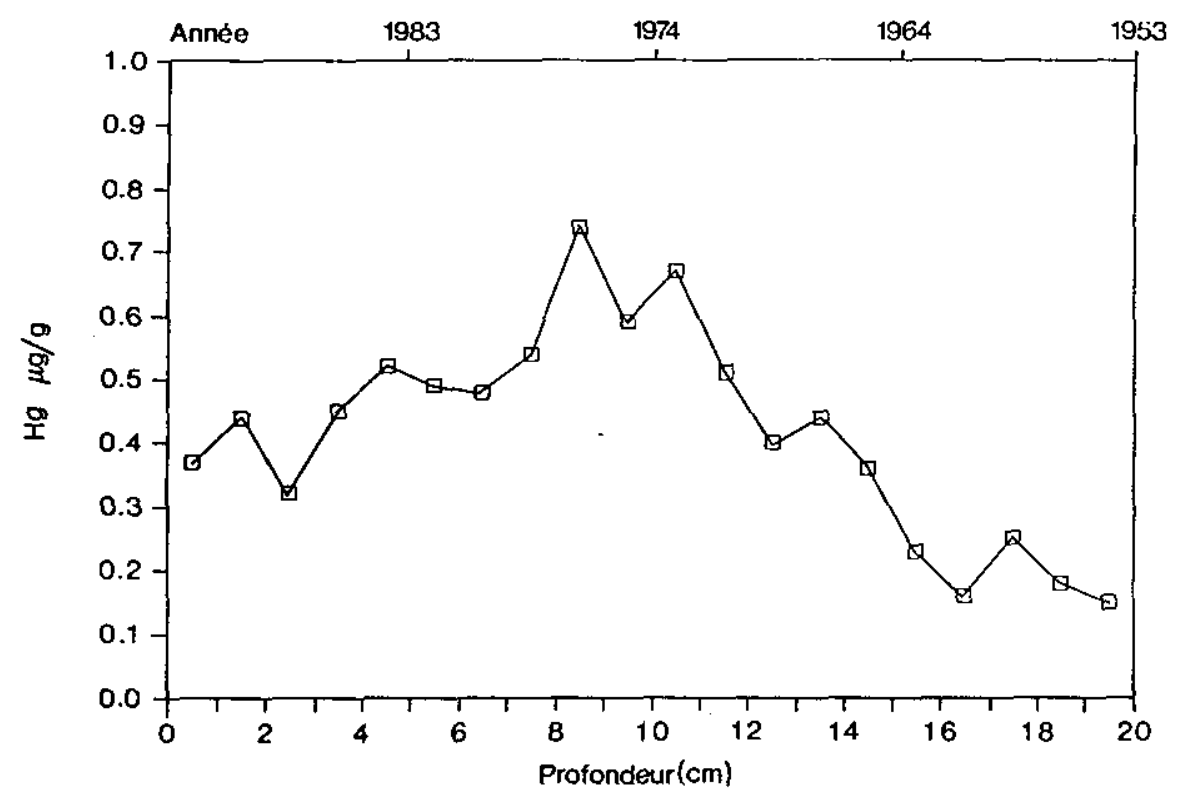

Flgure $7 \mathrm{a}$ Profils de concentration de $\mathrm{Hg}$ dans les sédiments de la station 3. Concentration profile of $\mathrm{Hg}$ in sediment from station 3.

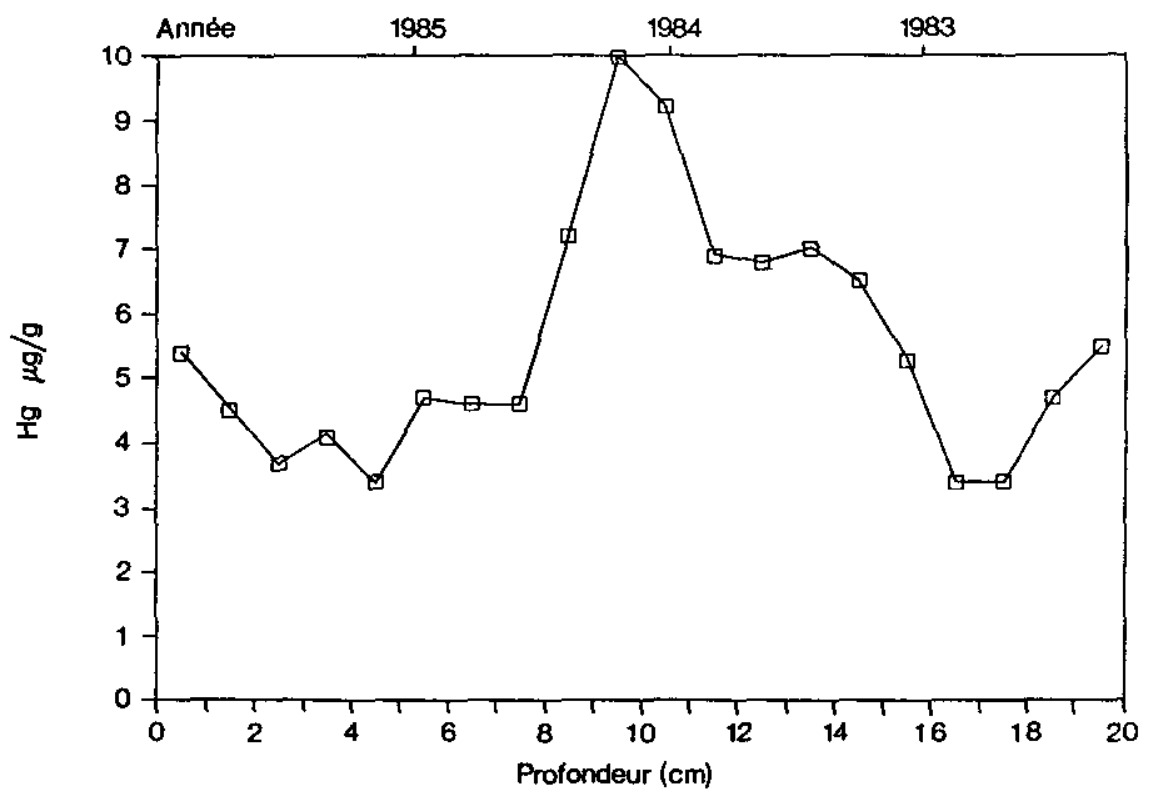

Figure $7 \mathrm{~b}$ Profils de concentration de $\mathrm{Hg}$ dans les sédiments de la station 21. Concentration profile of $\mathrm{Hg}$ in sediment from station 21. 
des particules de propriétés hydrodynamiques similaires transportées par le fleuve. Toutefois, on a noté un certain mélange de la couche de surface des sédiments à ces stations. Le profil de concentration de $\mathrm{Mn}$ dans les sédiments de la station 3 a été comparé à celui d'un bassin de dépót du lac Ontario (fig. 8). L'augmentation de $\mathrm{Mn}$ dans la couche de surface de $5 \mathrm{~cm}$ est due à la migration et a la précipitation de cet élément à la surface des sédiments dans la zone de dépôt relativement non perturbée du lac Ontario (GORHAM et SWAINE, 1965). On n'a pas observé une telle migration et accumulation de Mn à la surface du sédiment dans la zone de dépôt du lac Saint-Louis. Le mélange de la couche de surface des sédiments, plutôt que le régime anoxique à l'interface sédiment/eau, semble être responsable du manque de migration du Mn vers la surface des sédiments.

Figure 8 Profils de concentration de Mn dans les sédiments du bassin de dépót du lac Ontario et de la station 3 du lac Saint-Louis.

Concentration profiles of $\mathrm{Mn}$ in sediment from depositional basin in Lake Ontario and from station 3 in lake St. Louis.

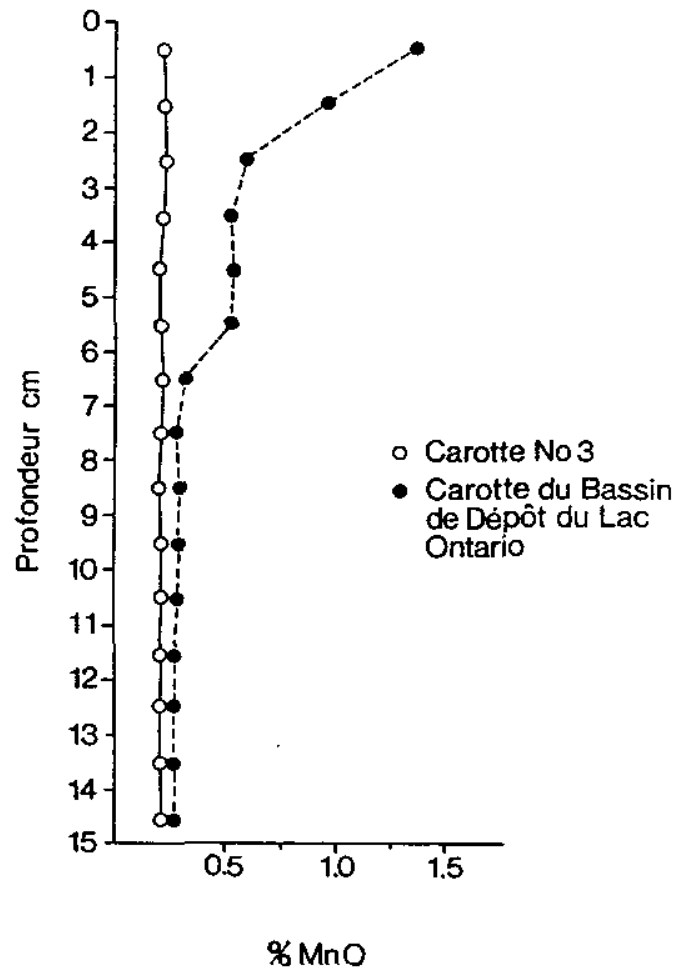

Dans les bassins de dépôts du lac Ontario, les concentrations en carbone organique ont augmenté de deux à trois fois vers la surface du sédiment. Les profils de concentration du carbone organique aux stations 3 et 21 du lac Saint-Louis ont montré une très faible accumulation de matière organique dans les sédiments de surface. L'augmentation plus importante de carbone organique dans les $5 \mathrm{~cm}$ de sédiment de surface à la station 21 , comparativement à la station 3 , peut être attribuée au dépôt de sédiments plus récents (1984-1985) contenant moins de matière organique décomposée que dans les sédiments âgés d'approximativement huit ans de la station 3 . 
Le $\mathrm{Zn}$ et le $\mathrm{Hg}$ étaient les deux seuls métaux présentant des variations dans les profils de concentration. Une augmentation de l'apport en $\mathrm{Zn}$ dans les 30 dernières années a été observée aux stations 3 et 9 . D'un autre côté, une diminution de ce même apport est survenue récemment à la station 17 . Une charge uniforme en $\mathrm{Zn}$ a été observée pour les cinq dernières années à la station 21. Les profils de concentration dans les sédiments du lac SaintLouis reflètent probablement les modifications survenues à l'intérieur de quelques points d'entrée locaux de $\mathrm{Zn}$ au lac. Les concentrations en $\mathrm{Hg}$ étaient, dans les sédiments de la station 21, d'un ordre de grandeur supérieur à celles des stations 3 et 9 . Une usine de chlore-alcali située sur la rive sud du lac Saint-Louis est une importante source ponctuelle de $\mathrm{Hg}$ dans les sédiments de la station 21 et dans la partie sud du lac (SLOTEROIJK, 1983; JARRY et al., 1985). Par conséquent, les fluctuations des concentrations en $\mathrm{Hg}$ dans les carottes de sédiment de la station 21 représentent des événements locaux survenus à la source ponctuelle. Les résultats ont indiqué un apport continu de $\mathrm{Hg}$ provenant de la rive sud du lac Saint-Louis. D'un autre côté, le profil de concentration $\mathrm{du} \mathrm{Hg}$ dans les échantillons de la station 3 était semblable à ceux observés dans les sédiments du lac Saint-Clair (MUDROCH et HILL, 1987) et du lac Ontario (MUDROCH, 1983), montrant une baisse dans la charge de $\mathrm{Hg}$ durant les 15 dernières années. Cette diminution correspond à l'atteinte d'un meilleur contrôle des rejets de mercure et à l'utilisation d'une nouvelle technologie aux usines de chlore-alcali situées à Sarnia, Ontario, et à Niagara Falls, état de Now York. La source de la rive sud du lac Saint-Louis pourrait contribuer à la contamination par le $\mathrm{Hg}$ des sédiments d'autres zones de dépôt du lac. Toutefois, les courants du lac empéchent le transport éventuel des sédiments contaminés par le $\mathrm{Hg}$ vers la station 3 située dans la partie nord du lac (fig. 1). L'augmentation de la charge en $\mathrm{Hg}$ à cette station après 1964 indique des apports en $\mathrm{Hg}$ d'autres sources le long du fleuve SaintLaurent, telles que des usines de chlore-alcali et de pâtes et papier (Ministère de l'Environnement de l'Ontario, 1977).

La concentration en métaux dans approximativement $5 \mathrm{~cm}$ à $7 \mathrm{~cm}$ de sédiments de surface du lac Saint-Louis est comparée, au tableau 1, à celles du lac Ontario, de la rivière des Outaouais et du fleuve Saint-Laurent, à environ $3 \mathrm{~km}$ en amont du lac Saint-Louis. Les concentrations en $\mathrm{Cu}, \mathrm{Cr}, \mathrm{Co}$, $\mathrm{Ni}, \mathrm{Pb}, \mathrm{V}, \mathrm{Zn}$ et $\mathrm{Hg}$ étaient plus élevées dans les sédiments du lac Saint-Louis que dans ceux des sédiments recueillis en amont dans le fleuve Saint-Laurent et dans la rivière des Outaouais. Les concentrations en $\mathrm{Cu}$ et en $\mathrm{Ni}$ étaient plus faibles dans les sédiments du lac Saint-Louis que celles mesurées dans les sédiments récents du lac Ontario. De même, les concentrations en $\mathrm{Pb}$ étaient plus faibles dans les sédiments de surface du lac Saint-Louis comparativement à celles du lac Ontario; toutefois, elles étaient plus élevées que celles des niveaux naturels de ce même lac Ontario. Des concentrations similaires en Zn ont été trouvées dans les sédiments de surface du lac Ontario et du lac Saint-Louis. Ces concentrations étaient cinq à six fois supérieures à celles mesurées pour le niveau de concentration naturel du lac Ontario. Les concentrations en $\mathrm{Cr}$, Co et $\mathrm{B}$ des sédiments de surface des lacs Ontario et Saint-louis étaient semblables à celles des sédiments datant d'avant la colonisation et obtenues à partir du lac Ontario. Ces métaux proviennent principalement des matériaux associés à la géologie régionale des Grands 
Tableau 1 Concentrations en métaux des sédiments de surface du lac Ontario, du fleuve Saint-Laurent et de la rivière des Outaouais ( $\mu \mathrm{g} / \mathrm{g}$ poids sec).

Table 1 Concentration of metals in surface sediments of Lake Ontario and St. Laxrence and Ottawa Rivers ( $\mu \mathrm{g} / \mathrm{g} d r y$ weight).

\begin{tabular}{|c|c|c|c|c|c|c|c|c|}
\hline & Cu & $\mathrm{Cr}$ & Co & $\mathbf{N}$ & $\mathrm{Pb}$ & $v$ & $2 n$ & $\mathrm{Hg}$ \\
\hline \multicolumn{9}{|l|}{ Lac Ontario ${ }^{1}$} \\
\hline Bassin ouest & $87-142$ & $110-190$ & $20-31$ & $83-97$ & $170-240$ & $94-145$ & $310-580$ & $0,55-1,89$ \\
\hline Bassin central & $74-115$ & $85-140$ & $11-20$ & $75-88$ & $120-190$ & $70-96$ & $390-545$ & $0,62-2,47$ \\
\hline Bassin est & $68-110$ & $86-136$ & $13-22$ & $76-92$ & $136-210$ & $77-103$ & $338-380$ & $0,65-3,12$ \\
\hline Coucha intérieure de sédiment* & $44-55$ & $90-105$ & $20-25$ & $56-65$ & $19-27$ & $135-145$ & $120-135$ & $0,20-0,30$ \\
\hline \multicolumn{9}{|l|}{ Rivière des Outaouais } \\
\hline Lac Des-Delax-Montagnes ${ }^{2}$ & $13-28$ & $49-147$ & $1-27$ & $29-57$ & $20-41$ & $78-124$ & $83-257$ & $0,11-0,50$ \\
\hline \multicolumn{9}{|l|}{ Fleuve Saint-Laurent ${ }^{3}$} \\
\hline $3 \mathrm{~km}$ en amont du lac Saint-Louis & $22-43$ & $100-103$ & $11-18$ & $47-52$ & $15-40$ & $75-77$ & $125-213$ & $0,39-0,50$ \\
\hline \multicolumn{9}{|l|}{ Lac Saint-Louis } \\
\hline Station 3 & $25-34$ & $116-118$ & $9-10$ & $58-59$ & $48-53$ & $115-121$ & $430-570$ & $0,32-0,52$ \\
\hline Station 17 & $26-30$ & $81-90$ & $13-14$ & $51-57$ & 53-54 & $88-96$ & $401-636$ & NA \\
\hline Station 21 & $36-40$ & $93-104$ & $14-17$ & $59-61$ & $45-50$ & $93-109$ & $288-320$ & $3,4-5,4$ \\
\hline
\end{tabular}

* Sédiments d'avant la colonisation ou niveau naturel.

1 Thomas et Mudroch (1979).

2 Neuf échantillons de sédiment de surface (Mudroch, données non publieses, 1986).

3 Quatre échantillons de sédiment de surface (Mudroch, données non publiées, 1986)

NA $=$ Non analysé. 
Lacs inférieurs (THOMAS et MUDROCH, 1979). Des concentrations accrues en $\mathrm{Hg}$ dans les sédiments de surface du lac Saint-Louis ont pour origine une usine de chlore-alcali, et ces concentrations étaient plus élevées dans certaines zones que celles des sédiments de surface du lac Ontario.

La relation entre les différentes tailles des particules de sédiment et les concentrations en métaux du lac Saint-Louis est donnée à la figure 9. Chaque fraction de taille de particule contenait une concentration différente de métaux spécifiques. En conséquence, les concentrations en métaux dans les sédiments seront différentes dans une zone d'accumulation de particules de taille $<13 \mu \mathrm{m}$ que, par exemple, dans une zone où la majorité des particules présentent une taille variant entre $18 \mu \mathrm{m}$ et $38 \mu \mathrm{m}$. La concentration la plus élevée en métaux dans les sédiments d'un bassin de dépôt d'un lac est habituellement associée à des particules de taille $<2 \mu \mathrm{m}$ (MUDROCH, 1984). Toutefois, seulement $21 \%$ à $46 \%$ des particules de sédiment avaient une taille de $2 \mu \mathrm{m}$ aux stations 3, 9, 17 et 21 du lac Saint-Louis, indiquant que ces zones sont des zones d'accumulation de particules de taille surtout comprise entre $2 \mu \mathrm{m}$ et $63 \mu \mathrm{m}$. De plus, ces zones ne constitueraient que des réservoirs temporaires pour les particules de taille $<2 \mu \mathrm{m}$, particules qui seraient éventuellement remises en suspension et transportées vers laval. Par conséquent, les petites particules présentant de plus fortes concentrations en métaux seront transportées dans une région où les conditions hydrodyna-

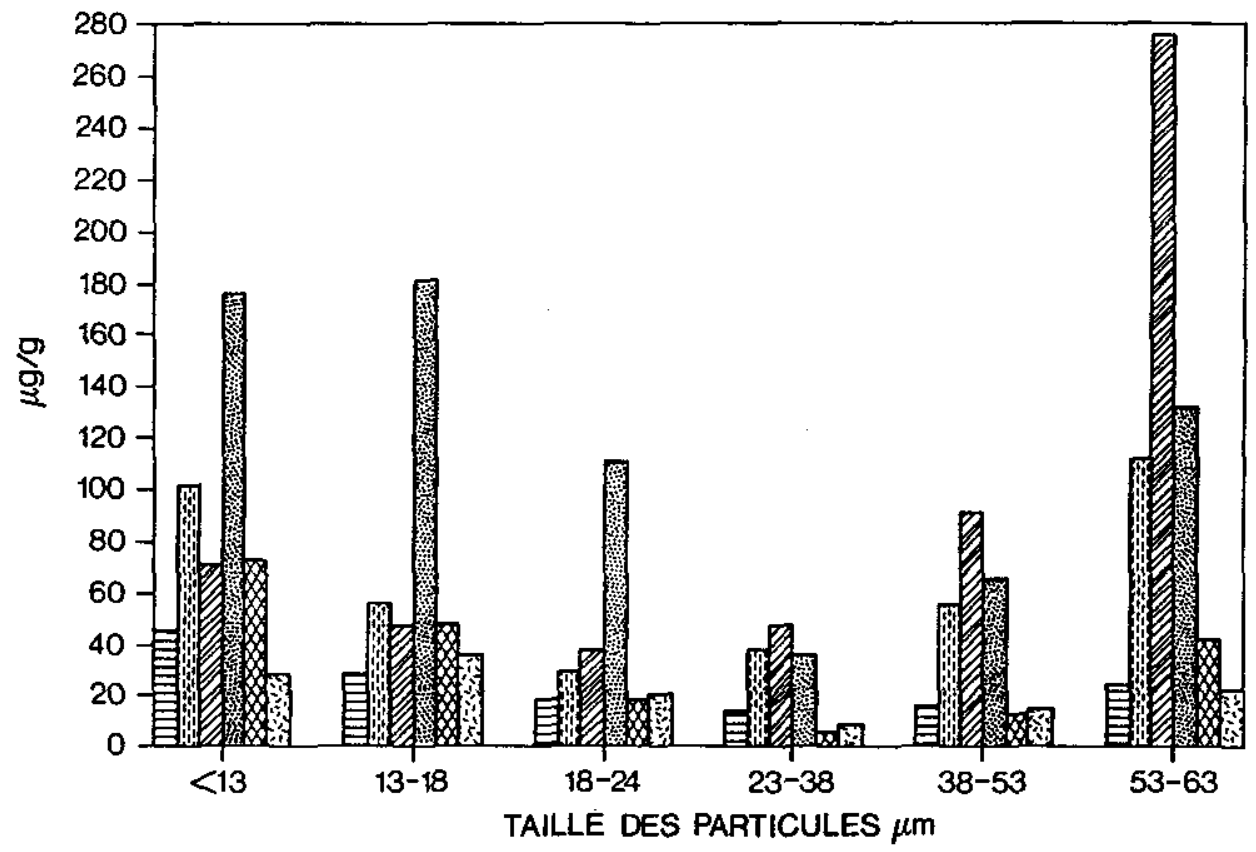

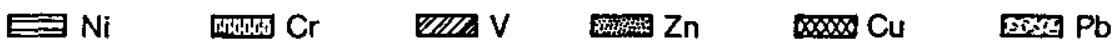

Flgure 9 Concentrations en métaux dans différentes fractions de taille des particules de sédiment de surface à la station 9 du lac Saint-Louis.

Concentrations of metals in different sediment particle size fractions in surface sediments at station 9 in Lake St. Louis. 
miques permettront leur sédimentation, cette région pouvant être l'estuaire du fleuve Saint-Laurent. Ces observations sont appuyées par l'épaisseur relativement petite de la couche de sédiment récent dans les zones de dépôt du lac Saint-Louis. Le sédiment résiduel dans ces zones est formé de grains ayant une densité, une cohésion et une taille suffisamment élevées pour que celui-ci ne puisse être déplacé par le régime énergétique local. Des concentrations accrues de quelques métaux dans le sédiment du lac Saint-Louis, en particulier le $\mathrm{Cr}$ et le $\mathrm{V}$ dans la fraction de particules dont la taille varie entre $53 \mu \mathrm{m}$ et $63 \mu \mathrm{m}$, peuvent être dues à la présence de particules de taille spécifique créées par lhomme et contenant de fortes concentrations de ces métaux. Une augmentation similaire du contenu en métaux a été observée dans la fraction limoneuse des sédiments des rivières Détroit et Niagara (MUDROCH, 1985 ; MUDROCH et DUNCAN, 1986).

\section{CONCLUSIONS}

1. Environ 20 à $30 \mathrm{~cm}$ de sédiment récent et fin (taille des grains $<63 \mu \mathrm{m}$ ) s'est accumulé dans quatre régions du lac Saint-Louis, dans le fleuve Saint-Laurent. Dans deux de ces régions situées dans la partie nord du lac, le taux de sédimentation était de 207 et $296 \mathrm{mg} \mathrm{cm}^{-2} \mathrm{a}^{-1}$, et l'âge d'une couche de $20 \mathrm{~cm}$ de sédiment était approximativement de 30 ans. Dans les deux autres régions, le long de la rive sud du lac, le taux de sédimentation était de 1837 et $2912 \mathrm{mg} \mathrm{cm}^{-2} \mathrm{a}^{-1}$, et la couche de sédiment de $20 \mathrm{~cm}$ d'épaisseur s'est déposée durant les cinq dernières années.

2. Environ $21 \%$ à $46 \%$ des particules de sédiment dans les carottes recueillies dans ces quatre régions étaient de taille $<2 \mu \mathrm{m}$, et $54 \%$ à $77 \%$ étaient de taille $<8 \mu \mathrm{m}$. Les profils de concentration des éléments majeurs et de la plupart des métaux étudiés dans les carottes de sédiment ont révélé un apport continu de matière similaire au point de vue géochimique. Une distribution uniforme de la taille des particules à travers les carottes montré que ces régions sont des zones de sédimentation pour les particules présentant des propriétés hydrodynamiques voisines et transportées par le fleuve SaintLaurent.

3. Les profils de concentration de $\mathrm{Hg}$ et de $\mathrm{Zn}$ dans le sédiment ont indiqué un apport de ces métaux à partir de sources locales le long du lac Saint-Louis. Les concentrations en $\mathrm{Cr}, \mathrm{V}$ et $\mathrm{Co}$ dans les sédiments des zones de dépôt étaient comparables à celles des sédiments de surface ainsi qu'aux concentrations naturelles retrouvées dans les bassins de dépôt du lac Ontario. Les concentrations en $\mathrm{Cu}, \mathrm{Ni}$ et $\mathrm{Pb}$ étaient plus faibles que celles des sédiments de surface présents dans les bassins de dépôt du lac Ontario. Les concentrations observées des métaux étudiés dans les sédiments du lac Saint-Louis ont été attribuées au triage des particules, à leur transport et au dépôt des particules de la fraction limoneuse (taille des grains comprise entre $2 \mu \mathrm{m}$ et $63 \mu \mathrm{m})$, particules susceptibles de contenir de plus faibles 
concentrations en métaux que les particules de la fraction argileuse (taille des grains $<2 \mu \mathrm{m}$ ) trouvée dans les bassins de dépôt du lac Ontario. Par conséquent, le lac Saint-Louis ne procure qu'un emmagasinement temporaire pour les métaux associés aux particules de la fraction argileuse des sédiments, particules qui sont éventuellement remises en suspension puis transportées vers l'aval.

\section{REMERCIEMENTS}

Les auteurs aimeraient remercier les membres du personnel de la section Opérations techniques, de l'Institut national de recherche sur les eaux, pour le prélèvement des carottes de sédiment dans le lac Saint-Louis.

\section{RÉFÉRENCES BIBLIOGRAPHIQUES}

ALLAN R.J., 1986. The limnological units of the lower great Lakes-St. Lawrence River corridor and their role in the sourceand aquatic fate of toxic contaminants. Water Poll. Res. J. Canada 21 (2), 168-186.

ALLAN R.J., 1987. Toxic chemical pollution of the St. Lawrence River and its upper estuary. Proc. Conf. on Coastal and Estuary Pollution IAWPRC/JAWPRI, Fukuoka, Japan, pub. Kyushe University, 102-113.

DUNCAN G.A., LAHAIE G.G., 1979. Size analysis procedure used in the sedimentology laboratory. Hydraulics Division, Manual, National Water Reasearch Institute, Burlington, Ontario.

GOLDBERG E.D., 1963. Geochronology with Pb-210. In : Radioactive Dating. International Atomic Energy Agency, Vienna, 121-131.

GORHAM E., SWAINE D.J., 1965. The influence of oxidizing and reducing conditions upon the distribution of some elements in lake sediments. Limnol. Ocea. nogr. 10, 268-279.

JARRY V., ROSS P., CHAMPOUX L., SLOTERDIJK H., MUDROCH A., COUIL-
LARD Y., LAVOIE F., 1985. Répartition spatiale des contaminants dans les sédiments du lac Saint-Louis (Fleuve SaintLaurent). Water poll. Res. J. Canada 20 (2), 75-99.

JOSHI S.R., 1987. Nondestructive determination of lead-210 and radium-226 in sediments by direct photon analysis. J. Padioanal. Nuct. Chem., Articles 116 (1), 169-182.

JOSHI S.R., SHUKLA B.S., MCNEELY R., 1988. The calculation of lead-210 dates for McKay Lake sediments. J. Radioanal. Nuct. Chem., Articles 125 (2), 341-349.

MAWHINNEY M.R., BISUTTIC., 1987. Common corers and grab samplers operating manual. Report, Technical Operations Section, National Water Research institute. Burlington, Ontario, Canada.

MUDROCH A., 1983. Distribution of major elements and metals in sediment cores from the Westem Basin of Lake Ontario. J. Great Lakes Res. 9, 125-133.

MUDROCH A., 1984. Particle size effect on concentration of metals in Lake Erie bottom sediments. Water Poll. Res. J. Canada 19, 27-35. 
MUDROCH A., 1985. Geochemistry of the Detroit River sediments. J. Great Lakes Res. 11, 193-200.

MUOROCH A., DUNCAN G.A., 1986. Distribution of metals in different size fractions of sediment from the Niagara River. J. Great Lakes Res. 12, 117-126.

MUDROCH A., HILL K., 1987. Distribution of mercury in Lake St. Clair sediments. National Water Reasearch Institute Contribution No 87-51, National Water Reasearch Institute, Burlington, Ontario, Canada.

MUDROCH A., KOKOTICH E., 1987. Determination of mercury in lake sediments using a gold film mercury analyzer. Analyst 112, 709-710.

OLDFIELD F., APPLEBY P.G., 1984. Empirical testing of $\mathrm{Pb}-210$ dating models for lake sediments. In: Lake Sediments and Environmental History, E.Y. Haworth and J.W.G. Lund (Eds.), Univ. of Minnesota Press, Minneapolis.

Ontario Ministry of the Environment, 1977. Concentrations of mercury in sediment and fish in the St. Lawrence River 1975. Ontario Ministry of the Environment, Southeastem Region.
PAGEAU G., 1971. La valeur des indices physico-chimiques dans la distinction des trois masses d'eau du lac Saint-Louis. Québec, Ministère du Tourisme, de la Chasse et de la Peche, Service de la faune. Rapport 6, 1-14.

PRÉFONTAINE G., 1942. Etude biologique des eaux de la plaine de Montréal et description générale des eaux de la plaine de Montreal. Rapport, Station Biol. Montréal, Inst. Biol. Univ. Montréal, fascicule 1, 33-67.

RUKAVINA N.A., 1986. Lac St. Louis bottom sediment data, Interim Report. Hydraulics Division, National Water Research Institute, Burlington, Ontario.

SLOTERDIJK H., 1983. St. Lawrence River Cornwall - Quebec City. Présentation au Water Quality Board Meeting, IJC (Commission mixte internationale), Québec, août 1983.

THOMAS R.L., MUDROCH A., 1979. Smalt Craft Harbours - sediment survey. Lakes Ontario, Erie and Lake St. Claire, 1978. Dredging Summary and Protocol. Fisheries and Oceans, The great Lakes Biolimnology Laboratory Report to Small Cartt Harbours, Ontario Region, p. 148. 